\title{
Exciton-thermal analysis of nonisothermal nucleation in solid solution of $\mathrm{CuCl}$ in glass
}

\author{
V.I. Leiman*, A.L. Ashkalunin, O.Yu. Derkacheva, and V.M. Maksimov \\ St. Petersburg State University of industrial technologies and design, 198095 St. Petersburg, Russia
}

\begin{abstract}
The work presents results of investigation of nucleation of $\mathrm{CuCl}$ nanocrystals in glass by method of exiton-thermal analysis (ETA) which is based on measuring the optical absorbance of $\mathrm{CuCl}$ phase in sample under heating up to the melting temperature of $\mathrm{CuCl}$ nanocrystals. The melting temperature of nanoparticle depends on its radius. Therefore analysis of curve of melting $\mathrm{CuCl}$ phase allows to determine distribution curve of nanoparticles on their radii. By the method of ETA the features of variation of $\mathrm{CuCl}$ nanoparticles distributions in glass when changing regime of sample annealing (nonisothermal nucleation) were studied. For example, by reducing the heating rate of the sample the concentration of particles of $\mathrm{CuCl}$ phase increases considerably while reducing average radius.
\end{abstract}

Method of exiton-thermal analysis (ETA) of melting-crystallization of $\mathrm{CuCl}$ nanoparticles in glass is based on application of the well known Urbach's rule [1]. The rule reads: the long-wavelength absorption edge of crystals at different temperatures intersects in one "node point". The Urbach's rule is true for most of transparent media [2].

If you heat the sample and transmit light with energy of "node point" photon through the sample of glass with $\mathrm{CuCl}$ nanocrystals $(\mathrm{NC})$, then absorbance $\mathrm{NC} \mathrm{CuCl}$ does not change until beginning $\mathrm{NC}$ melting. Melting $\mathrm{CuCl} \mathrm{NC}$ leads to transition of the nanoparticles in the amorphous state and the exciton states, which ensure absorption in the "nodal point", disappear. But zone-zone absorption of $\mathrm{CuCl}$ nanomelt remains. This absorption is almost two times less than nanocrystals one. The temperature of melting $\mathrm{CuCl}$ $\mathrm{NC}$ decreases with decreasing particles radius. Since NC distribution of radius takes a place in the glass, first the smallest $\mathrm{CuCl}$ particles of the distribution are melted, and then with increasing temperature the nanocrystals of bigger radii are melted. Thus, there is an optical scanning melting distribution of $\mathrm{CuCl} \mathrm{NC} \mathrm{[3].}$

As a result, we have experimental curve of melting $\mathrm{CuCl}$ nanoparticles - dependence of optical absorption coefficient of the sample on temperature $K_{0}(T)$. Derivation $K_{0}(T) / d T$ is intensity of melting $\mathrm{CuCl}$ phase in dependence on sample temperature at linearly increasing temperature. Since melting NC of determined radius takes place at corresponding temperature, it is possible to obtain from the function $K_{0}(T) / d T$ the distribution of $\mathrm{CuCl}$ phase in sample on radii - the curve $f_{V}(r)=d V / d R$.

\footnotetext{
${ }^{*}$ Corresponding author: leiman@inbox.ru
} 
Because ETA method is based on optical absorption it does this method very sensitive to investigate the $\mathrm{CuCl}$ nanocrystals in glass. ETA method allows to carry out studying $\mathrm{CuCl} \mathrm{NC}$ with sizes from 1 to $30 \mathrm{~nm}$ and resolution up to $0.1 \mathrm{~nm}$.

This method was applied to investigate features of process of nonisothermal nucleation of $\mathrm{CuCl}$ phase in glass at different rate of heating glass sample. It has been shown that a significant increasing the concentration of the $\mathrm{CuCl}$ phase is observed at decelerated heating (curves 1-6) sample compared with the rapid heating (curves 7-9). During slow heating the sample there is a maximum concentration of the $\mathrm{CuCl}$ phase in the 40th minute (curve 2). With the continuation of the nucleation (solution depletion) and the growth of temperature the critical radius increases, so that dissolving portion of previously formed $\mathrm{CuCl}$ clusters begins [4]. Therefore, there is a reduction $\mathrm{CuCl}$ phase concentration (curves 3-6).

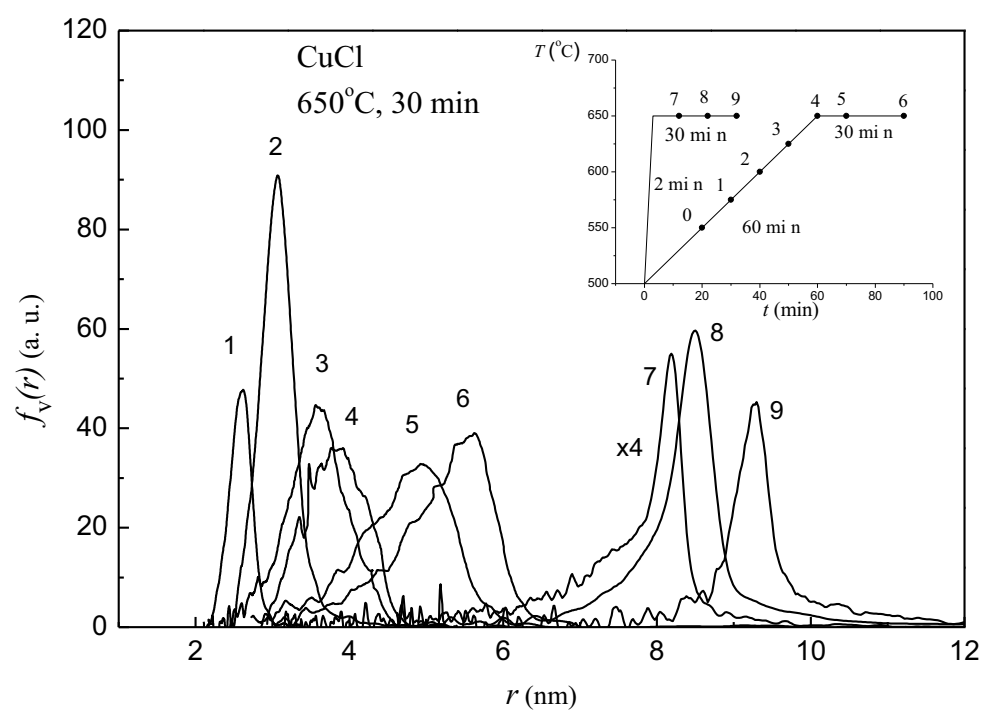

Fig. 1. Change in the distribution of the $\mathrm{CuCl}$ phase volume on the radius of the particle at slow heating (curves 1-6) and rapid heating (curves 7-9) the samples up to a temperature of $650{ }^{\circ} \mathrm{C}$ and holding for 30 minutes. Annealing time from the start of sample heating is shown in the inset by points and corresponds to the distribution number. The values of the curves 7-9 are multiplied by 4 times.

Thus, the first it is shown that at changing rate of heating a solid solution it should take into account the initial processes of nonisothermal nucleation which lead to a change in the sizes and concentration of new phase particles.

\section{References}

1. F. Urbach, Phys. Rev. 92, 1324 (1953)

2. M.V. Kurik, Phys. Stat. Sol. A 8, 9 (1971)

3. P.M. Valov, V.I. Leiman, Physics of the Solid State 51, 1703 (2009)

4. P.M. Valov, V.I. Leiman, V.M. Maksimov, O.Yu. Derkacheva, Physics of the Solid State 52, 821 (2010) 